\title{
Efficacy of Freeze-Dried Amnion Grafts on Cytokines in Uterine Exudates Following Hysteroscopic Adhesiolysis of Severe Intrauterine Adhesions
}

\author{
Sha Wang, Hua Duan, Bohan Li, Yiyi Wang, Zhengchen Guo, Xinyu Zhu \\ Minimally Invasive Gynecologic Center, Beijing Obstetrics and Gynecology Hospital, Capital Medical University, Beijing, I00006, People's Republic of \\ China \\ Correspondence: Hua Duan, Email duanhua@ccmu.edu.cn
}

Background: Intrauterine adhesions (IUAs) are a benign uterine disorder that results in intrauterine adhesions and scarring. This study was conducted to assess the effects of freeze-dried amnion grafts on the concentrations of adhesion-related cytokines in uterine exudates following hysteroscopic adhesiolysis of IUAs.

Materials and Methods: This study was a prospective randomized controlled trial. Thirty patients who underwent hysteroscopic adhesiolysis for severe IUAs were enrolled. They were randomly divided into a study group (with freeze-dried amnion graft treatment after surgery, $\mathrm{N}=15$ ) and a control group (without a graft, $\mathrm{N}=15$ ). After complete separation of adhesions, a Foley balloon containing a freeze-dried amnion graft was inserted into the uterine cavity in the study group, while a Foley balloon with no graft was placed in the control group. Enzyme-linked immunosorbent assays were performed to test adhesion-related cytokine concentrations in uterine exudates, including IL-1 $\beta$, TNF- $\alpha$, and VEGF, at different time intervals after surgery. Second-look hysteroscopy was conducted three months after the surgery. On the basis of the American Fertility Society (AFS) scoring system, the level of adhesions was evaluated during hysteroscopy.

Results: The postoperative volume of the uterine exudates in the study group was significantly lower than that of the control group $(P<0.05)$. The adhesion-related cytokine concentrations of TNF- $\alpha$, VEGF, and IL- $1 \beta$ significantly increased after surgery in both groups. Their concentrations were significantly lower in the study group than in the control group $(P<0.05)$. Moreover, IL-1 $\beta$ exhibited a long-lasting effect in the study group. AFS scores and readhesion rates were significantly lower in the study group than in the control group after hysteroscopic adhesiolysis of severe IUAs.

Conclusion: The application of freeze-dried amnion grafts is beneficial to the postoperative recovery of patients with severe IUAs and may reduce the readhesion rate after hysteroscopic adhesiolysis by lowering adhesion-related cytokines, including TNF- $\alpha$, VEGF, and IL-1 $\beta$.

Keywords: freeze-dried amnion graft, intrauterine adhesions, interleukin- 1 beta, tumour necrosis factor- $\alpha$, vascular endothelial growth factor

\section{Introduction}

Intrauterine adhesions (IUAs), also known as Asherman's syndrome, occur due to trauma to the basal layer of the endometrium from various causes, and the uterine anatomy is disrupted by intramural uterine adhesions. The uterine anatomy is disrupted by intramural uterine adhesions, resulting in hypomenorrhea and amenorrhea, infertility, recurrent pregnancy loss, and abnormal placenta implantation or placenta previa. ${ }^{1}$ Currently, the standard method of treatment for IUAs is transcervical resection of adhesions (TCRA). However, the prognosis of severe IUAs remains poor, with a recurrence rate of up to $62.5 \%$, even when the uterine cavity has been restored after hysteroscopic adhesiolysis, which seriously influences the efficacy of hysteroscopic adhesiolysis. ${ }^{2}$ Several methods have been proposed to prevent postoperative readhesions. Many ancillary treatments, such as the placement of an intrauterine device, intrauterine stents, or a 
balloon in the uterine cavity after surgery, the use of a gel containing antiadhesion substances, and oestrogen therapy during the postoperative period, have been applied to stimulate endometrial regeneration.

Previous studies found that under electric heating, isolated wounds exude fluid containing several cytokines, including interleukin-1 beta (IL-1 $\beta$ ), tumour necrosis factor- $\alpha$ (TNF- $\alpha$ ), and vascular endothelial growth factor (VEGF), after hysteroscopic adhesiolysis. ${ }^{3,4}$ These factors play important regulatory roles in injury repair. Notably, appropriate cytokine levels regulate the balance between the degradation and synthesis of extracellular matrix (ECM) and promote repair of the uterine cavity. Nevertheless, excess cytokine production disrupts this balance, causing excessive accumulation of ECM and the formation of IUAs. In recent years, freeze-dried amnion was reported to secrete a variety of biologically active factors that promote cell growth and improve the microenvironment by regulating cell differentiation and function. Moreover, freeze-dried amnion grafts exhibit antifibrotic, anti-inflammatory, antiangiogenic, and antimicrobial features, which promote tissue repair. ${ }^{5}$ As a result, these grafts are widely used in clinical specialties, including ophthalmology, dermatology, and orthopaedics, to prevent the formation of scars. ${ }^{6}$ In terms of IUAs, in some case studies, freeze-dried amnion grafts were applied to uterine cavity wounds after hysteroscopic adhesiolysis. ${ }^{7,8}$ Fresh amnions are associated with many issues, such as ethical concerns, quality control, preservation, and transportation. Our research group previously selected freeze-dried amnion grafts with similar biological activity to those applied in patients diagnosed with severe IUAs. Hysteroscopic reexamination and follow-up records showed that freeze-dried amnion grafts reduced adhesion scores and improved menstruation in patients with severe IUAs. ${ }^{8}$ However, the precise mechanisms and efficacy are still controversial.

Despite recent clinical studies on the application of freeze-dried amnion after hysteroscopic adhesiolysis, no consistent theoretical foundation has been established. Based on findings from previous clinical research, this study used freeze-dried amnion to cover uterine cavity wounds after hysteroscopic adhesiolysis and measured the amount of uterine exudates. Furthermore, we monitored changes in the concentrations of the adhesion-related cytokines TNF- $\alpha$ and VEGF and the repair-associated factor IL-1 $\beta$ in the exudates. Subsequently, the effects of freeze-dried amnion grafts on the amount of uterine exudate and repair of the endometrium were analysed. Furthermore, the mechanism of freeze-dried amnion grafts in preventing readhesion was explored to provide a theoretical foundation for clinical practice. Additionally, the duration of Foley balloon placement has not yet been standardized. Clinical treatment ranges from 3-7 d after surgery. No systematic studies have explored the duration of balloon placement. Therefore, we calculated the amount of uterine cavity exudate after surgery and explored the duration of early postoperative uterine cavity inflammation.

\section{Materials and Methods}

\section{Patients}

Based on the inclusion and exclusion criteria, this study selected patients who underwent intrauterine adhesiolysis for severe IUAs in Beijing Obstetrics and Gynecology Hospital affiliated with Capital Medical University between February 2016 and December 2017. The AFS scoring system was used to evaluate IUAs. ${ }^{9}$ The inclusion criteria were as follows: (1) age younger than 40 years; (2) hypomenorrhea or amenorrhea; (3) infertility or spontaneous abortion ( $\geq 1$ ); and (4) a previous AFS IUA score of at least eight. On the other hand, the exclusion criteria included (1) premature menopause; (2) existence of other intrauterine lesions (eg, polyps, myoma, or septa); and (3) severe intercurrent disease (eg, systemic disease, coagulative disorders, or severe disease of the kidneys or liver).

A total of 30 patients were randomized into either an amnion group or a control group in a 1:1 ratio using a computergenerated randomization sheet. Patients were randomly assigned by computer and processed by a third-party investigator. The trial was double-blinded to the subjects and clinicians and was unblinded after the hysteroscopic follow-up results. No significant difference was found between the two groups in age, gravidity and parity history, or preoperative AFS scores (Table 1). This study was a randomized clinical controlled trial (Trial Registration Number NCT02496052). Each participant in the study signed an informed consent form. The study was approved by the Research Ethics Committee of Beijing Obstetrics and Gynecology Hospital, where the experiments were performed and was performed in accordance 
Table I Baseline Characteristics of the Two Groups of Patients $(n=15)$

\begin{tabular}{|l|l|l|l|}
\hline Parameter & Study Group & Control Group & $P$ \\
\hline Age, mean (SD) & $28.9(1.7)$ & $29.8(2.1)$ & 0.208 \\
Gravidity, mean (SD) & $3.1(1.1)$ & $3.0(1.2)$ & 0.814 \\
Parity, median (range) & $0(0,1)$ & $0(0,1)$ & 0.426 \\
AFS, median (range) & $9(7,12)$ & $9(7,11)$ & 0.394 \\
\hline
\end{tabular}

with the Helsinki Declaration of 1975, as revised in 2008. All enrolled patients signed an informed consent form before surgery.

\section{Sterilized Freeze-Dried Amnion Grafts}

Thanks to Jiangxi Rui Ji Biotechnology (Jiangxi, China), we obtained the sterilized freeze-dried amnion grafts used in this study, and the production processes were described as follows. Fresh amniotic tissues were collected and freeze-dried at low temperature and then sterilized by cobalt-60 radiation.

\section{Sample Collection}

Under ultrasound guidance, continuous perfusion hysteroscopy using a resectoscope was used to perform hysteroscopic adhesiolysis. In the study group, two $30-\mathrm{mm} \times 20-\mathrm{mm}$ pieces of freeze-dried amnion were wrapped in the top of a Foley balloon and placed into the uterine cavity. The specific processing flow is shown in Figure 1. In the control group, only a Foley balloon was placed. The Foley catheter, with the tip distal to the balloon cut away, was used as a scaffold for insertion of the amnion graft into the uterine cavity. In the study group, the balloon portion of the Foley catheter was

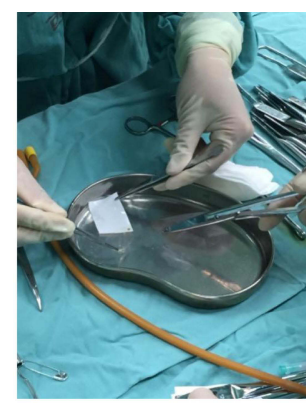

Amniotic membrane water-soaking

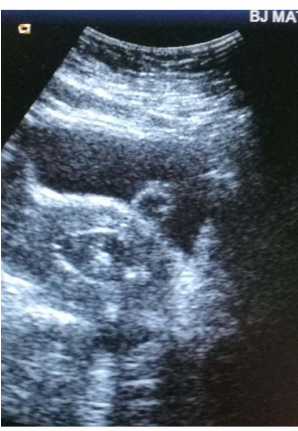

Ultrasonography

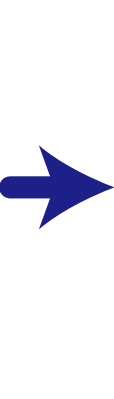

Top removing of Foley catheter balloon
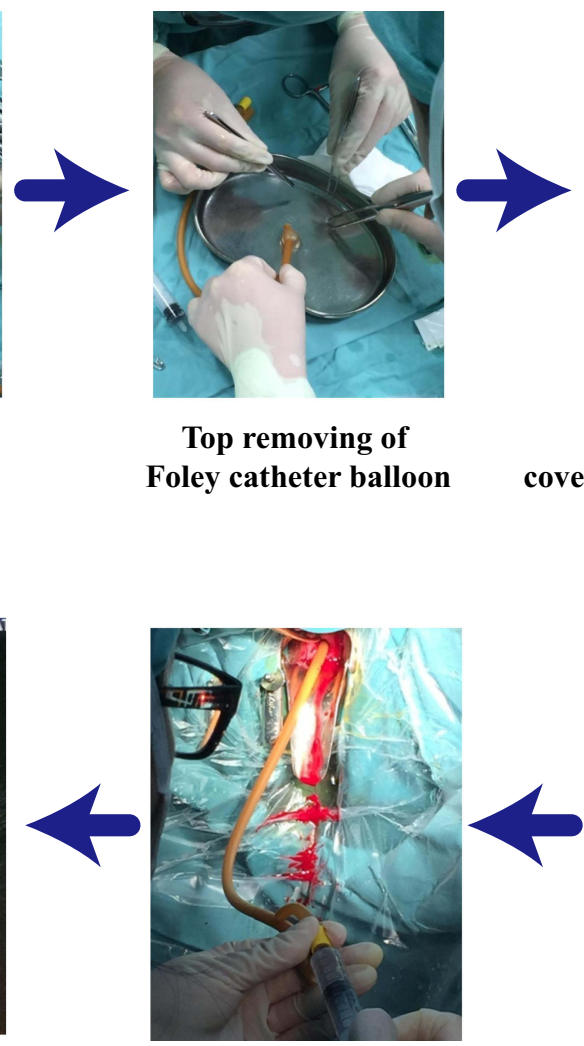

Balloon flooding
Foley catheter balloon

covered by amniotic membrane
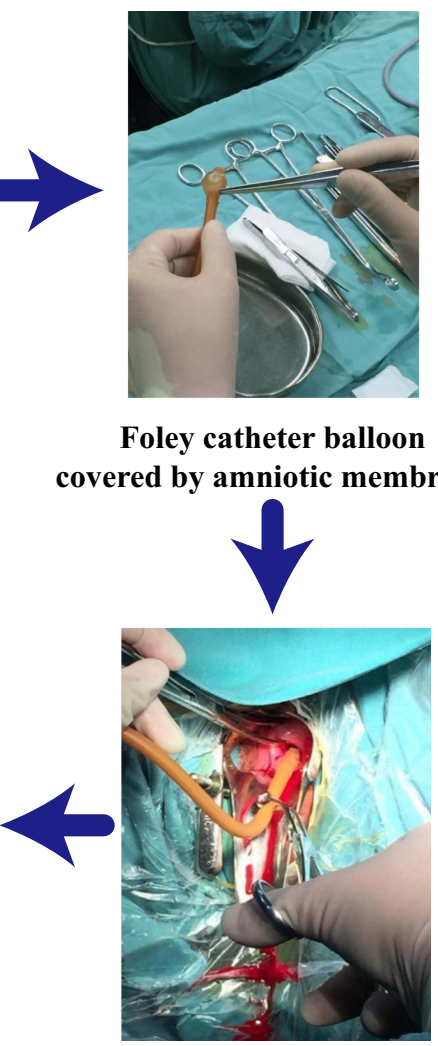

Inserting into uterine cavity

Figure I Processing flow of freeze-dried amnion grafts. 
covered with a sterilized freeze-dried amnion graft and hydrated in sterile normal saline for 10 min before use. Two amnion grafts were applied to the Foley catheter, with the epithelial amnion membrane surface facing outward. The Foley catheter was then inserted into the uterine cavity under ultrasonographic guidance. The balloon was initially inflated with $8-10 \mathrm{~mL}$ of normal saline for $2-3 \mathrm{~min}$ to ensure that the amnion graft fully adhered to the uterine cavity. Afterwards, $3-5$ $\mathrm{mL}$ of the normal saline solution was withdrawn, leaving an average of $5 \mathrm{~mL}$ within the balloon. In the control group, the protocol for insertion of the Foley catheter and inflation of the balloon was the same as that used in the amnion group; however, amnion grafting was not used. The Foley catheter remained in place for 1 week, after which time the balloon was deflated and the catheter was removed as an outpatient procedure.

Uterine exudates were collected at intervals of $3 \mathrm{~h}, 6 \mathrm{~h}, 9 \mathrm{~h}, 12 \mathrm{~h}, 24 \mathrm{~h}, 48 \mathrm{~h}$, and $72 \mathrm{~h}$ and $4 \mathrm{~d}, 5 \mathrm{~d}, 6 \mathrm{~d}$, and $7 \mathrm{~d}$ after surgery, and the total amount was measured at every time interval. Less than $4 \mathrm{~mL}$ of exudate was transferred into a vacuum centrifuge tube and immediately centrifuged at $3000 \mathrm{rpm}$ at room temperature for $15 \mathrm{~min}$, and then $300 \mu \mathrm{L}$ supernatant was taken and transferred to a cryotube and was stored at $-80^{\circ} \mathrm{C}$.

\section{Detection of Adhesion-Related Factor Concentration}

The concentrations of IL-1 $\beta$, TNF- $\alpha$, and VEGF in the uterine exudate collected at various time points after hysteroscopic adhesiolysis were determined by using human IL-1 $\beta$, TNF- $\alpha$, and VEGF enzyme-linked immunosorbent assay (ELISA) kits, respectively (USCN Life Science Inc., Wuhan, China), following the manufacturer's instructions. Then, 50 $\mu \mathrm{L}$ of sample or standard was added to each well, and $50 \mu \mathrm{L}$ of detection reagent was applied and incubated at $37^{\circ} \mathrm{C}$ for $30 \mathrm{~min}$. Subsequently, $50 \mu \mathrm{L}$ of colour development reagent A and $50 \mu \mathrm{L}$ of reagent B were added and incubated at $37{ }^{\circ} \mathrm{C}$ for $15 \mathrm{~min}$. Finally, $50 \mu \mathrm{L}$ of stop solution was added to each well, and the optical density (OD) was recorded at $450 \mathrm{~nm}$. In the course of the procedure, the plate was washed using a routine ELISA method. According to the Yolken RH standard, the linear regression equation of the standard curve was obtained through the use of the concentration and OD value of the standard substance. To calculate the actual cytokine concentration, we added the sample OD values to the equation to obtain the sample concentration in $\mathrm{ng} / \mathrm{L}$, which we then multiplied by the dilution factor. We collected and measured exudates from 15 patients at each time point, and the results of each group were the mean of three repeated measurements.

\section{Statistical Analyses}

Statistical analysis was performed by SPSS statistical software version 22.0 (IBM, NY). Quantitative values are expressed as the mean \pm standard deviation (SD). Moreover, one-way analysis of the $t$-test results was performed to compare differences within and between the groups. The results at different time points within the groups were compared by the Student-Newman-Keuls (SNK) test. The measurement data are expressed as the median (minimum valuemaximum value). A $P$ value $<0.05$ by the rank-sum test was considered statistically significant.

\section{Results}

\section{Volume Changes in Uterine Exudates}

The total volume of the uterine exudates is shown in Figure 2. First, an analysis of the total exudation volumes in the two groups after surgery was performed. In the study group, the total uterine exudate volumes were significantly lower than those in the control group $(P=0.003)$.

Then, the daily amounts of exudate were measured. The volume of uterine exudates in the study group began to decrease $48 \mathrm{~h}$ after surgery but was reduced $4 \mathrm{~d}$ after surgery in the control group. The peak values were significantly different between the two groups $(P=0.00)$.

In contrast to the peak value, the volumes of exudates in both groups decreased significantly after $7 \mathrm{~d}(P=0.000$ and $P=0.002$ for the study group and control group, respectively). The amount of uterine exudate in the study group was significantly lower than that in the control group $7 \mathrm{~d}$ postoperatively $(P=0.000)$. 


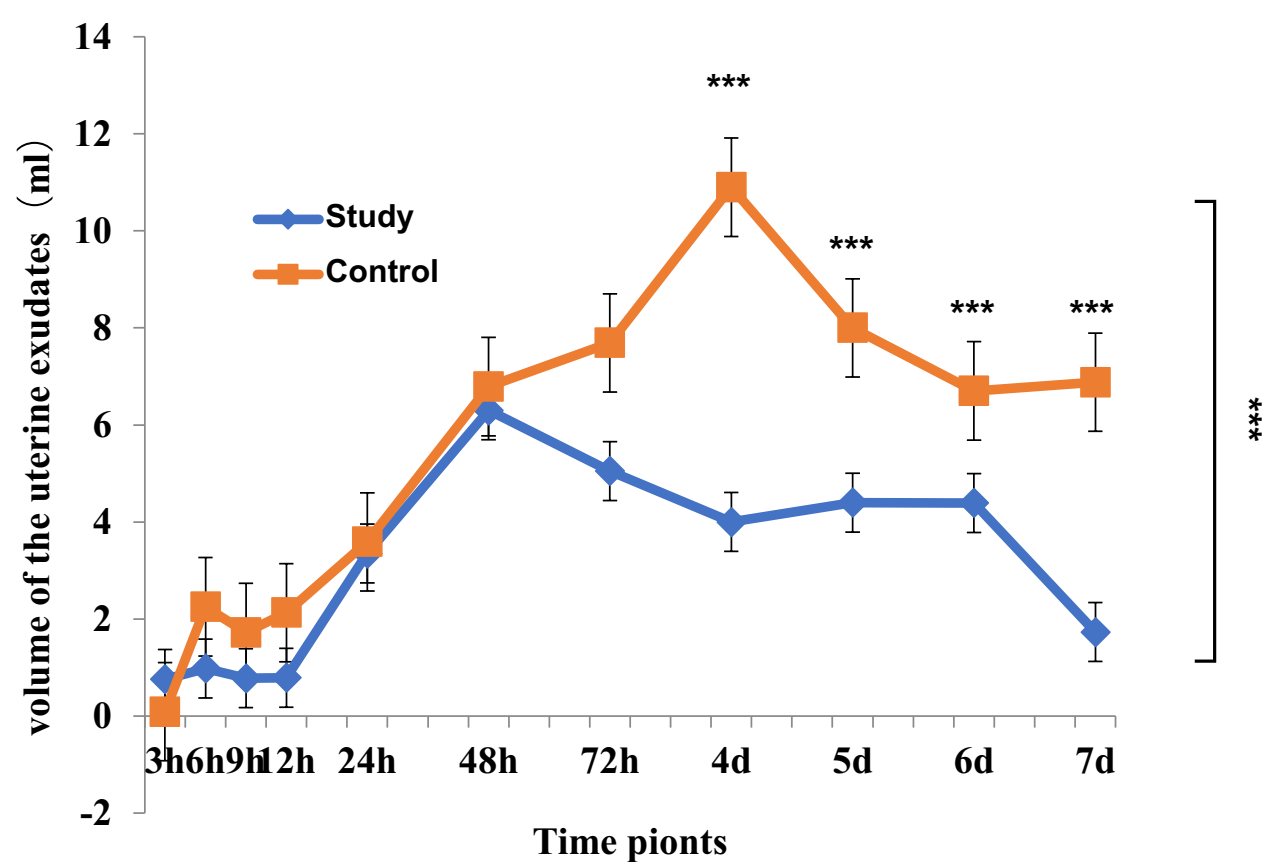

Figure 2 Changes in the volume of the uterine exudate with time. The volume of daily exudate and the total volume were compared. $* * * P<0.00 \mathrm{I}$.

\section{Cytokine Concentrations in Uterine Exudates}

After surgery, the average TNF- $\alpha$ concentration in the study group was significantly lower than that in the control group $(P=0.014)$.

As shown in Figure 3, the TNF- $\alpha$ concentration in the uterine exudate in the control group was significantly higher than that in the study group at every time point $(P<0.05)$. The TNF- $\alpha$ level in the uterine exudates from the two groups of patients first increased and then decreased. The peak TNF- $\alpha$ concentration in the exudates of the two groups was identified on the 2nd day after surgery and then gradually decreased. In the study group, a significant difference in TNF- $\alpha$ concentrations was found between the 7th day after surgery and the peak value $(P<0.05)$ compared to those in the control group $(P>0.05)$.

After surgery, the average VEGF concentration in the study group was significantly lower than that in the control group $(P=0.004)$. As shown in Figure 4, the VEGF concentration in the control group increased rapidly and peaked the day after surgery, while in the study group, the concentration peaked at $3 \mathrm{~d}$. Except for the $3 \mathrm{rd}$ and 4 th days postoperatively, the VEGF concentration in the study group was significantly lower than that in the control group $(P<0.05)$. On the 7th day after surgery, VEGF levels in the two groups were significantly lower than the peak value $(P<0.05)$.

Within $7 \mathrm{~d}$ after surgery, the average IL-1 $\beta$ concentration in the study group was significantly lower than that in the control group $(P=0.005)$.

Unlike the aforementioned cytokines, IL- $1 \beta$ concentrations peaked on the 6th day and 5th day after surgery in the study group and the control group, respectively. Nevertheless, the subsequent decrease was not significant $(P>0.05)$. Moreover, although the IL-1 $\beta$ expression level was significantly different between the study group and the control group at each time point $(P<0.05)$, the expression levels in the two groups seemed similar on the 7 th day after surgery $(P>0.05)$. The specific details are shown in Figure 5.

\section{Postoperative AFS Scores and Readhesion Rates}

We conducted hysteroscopy and reassessed the patients' AFS scores 3 months after their operation to observe the recovery of the endometrium in the patients. According to the results, we found a significant decrease in postoperative AFS scores in both groups, while the magnitude of the postoperative decrease was significantly different when compared 


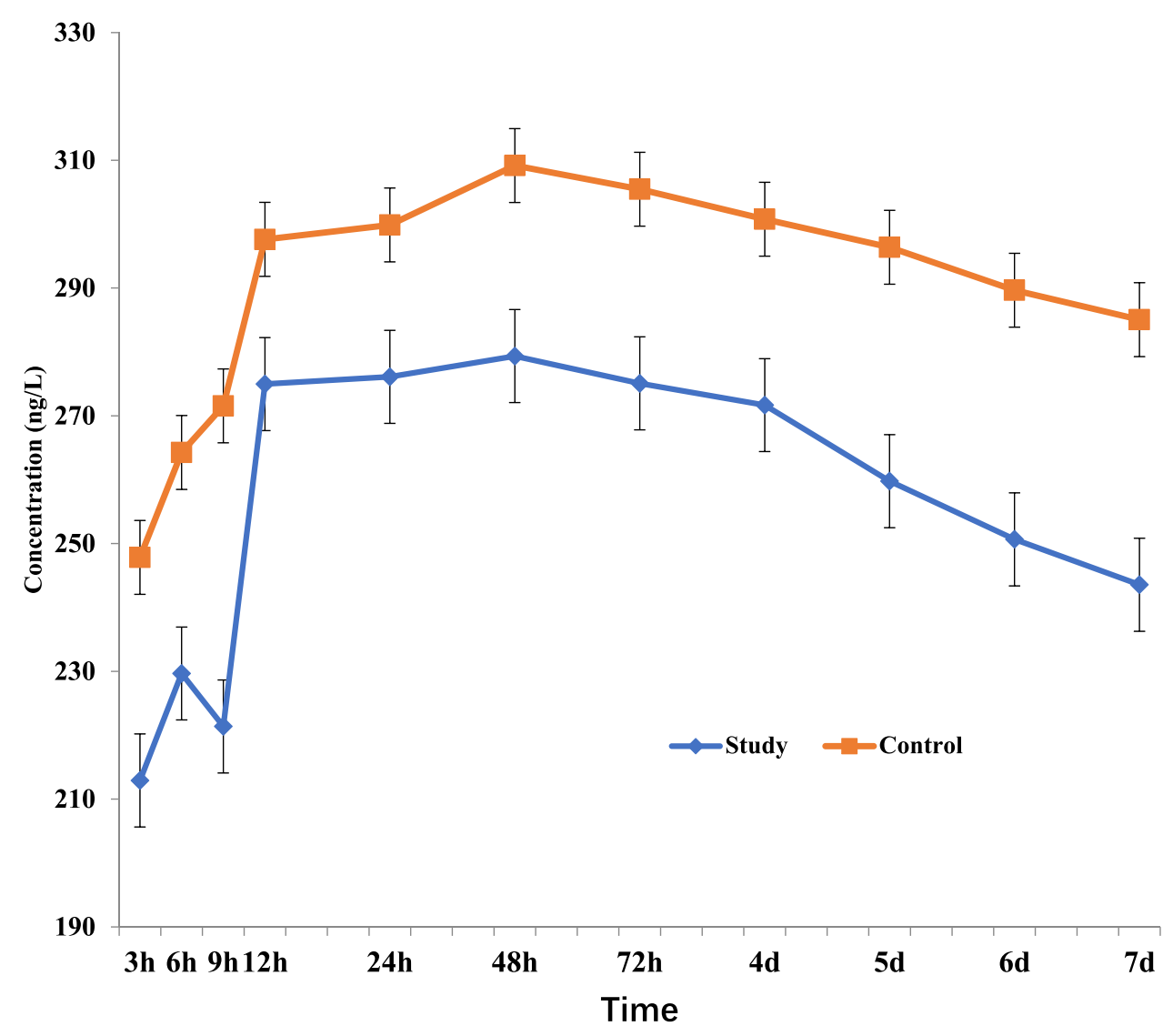

Figure 3 Changes in TNF- $\alpha$ in the uterine exudate with time. The concentration at each time point between the study and control groups was compared, as was the concentration between the peak and the 7th day after the operation.

between the two groups, as shown in Figure 6A. Based on the scoring results, we defined postoperative readhesion as an AFS $>5$ and calculated the postoperative readhesion rate for both groups separately. Likewise, a significant difference in the rate of postoperative readhesion was found between the two groups, as shown in Figure 6B.

No surgical complications, such as uterine perforation, fluid overload, severe bleeding, or infection, were observed in either group. Complication associated with insertion of the Foley balloon include falling off within $7 \mathrm{~d}$, fever, and abdominal pain. None of these Foley balloon-related complications were observed in either group.

\section{Discussion}

Hysteroscopic adhesiolysis is a type of plastic surgery performed on the uterine cavity under direct hysteroscopic visualization to resect scar tissue and restore anatomical morphology. Notably, endometrial damage is extensive in severe cases of IUAs; therefore, surgery emphasizes effective protection of the residual endometrium. However, the residual endometrial area is less than $1 / 3$ the area of the uterine cavity, causing a large wound after surgical resection. ${ }^{1}$ In addition, due to electrical intervention during the operation, the high-frequency electric action of the electrode inevitably produces a particular electrical heating effect on healthy endometrial tissue and uterine muscle wall tissue, simultaneously separating and removing scar tissue in the uterine cavity. ${ }^{4}$ Nevertheless, the role of high-frequency electric hysteroscopic treatment during separation and correction surgery for severe IUAs is still very important. Moreover, the unique anatomical morphology of the uterine cavity is a factor in wound exudation after hysteroscopic adhesiolysis surgery and in the action of cytokines on surgical wounds. Wound exudates are known to contain many repair- and adhesion-related factors. Partial cytokines might activate the proliferation of mesenchymal cells (mainly fibroblasts), thereby causing deposition of large amounts of fibrinogen, fibronectin, and other substances to form fibrin scaffoldcovered wounds, resulting in excess ECM accumulation. Inflammatory granulation tissue and fibrous scars are formed, 


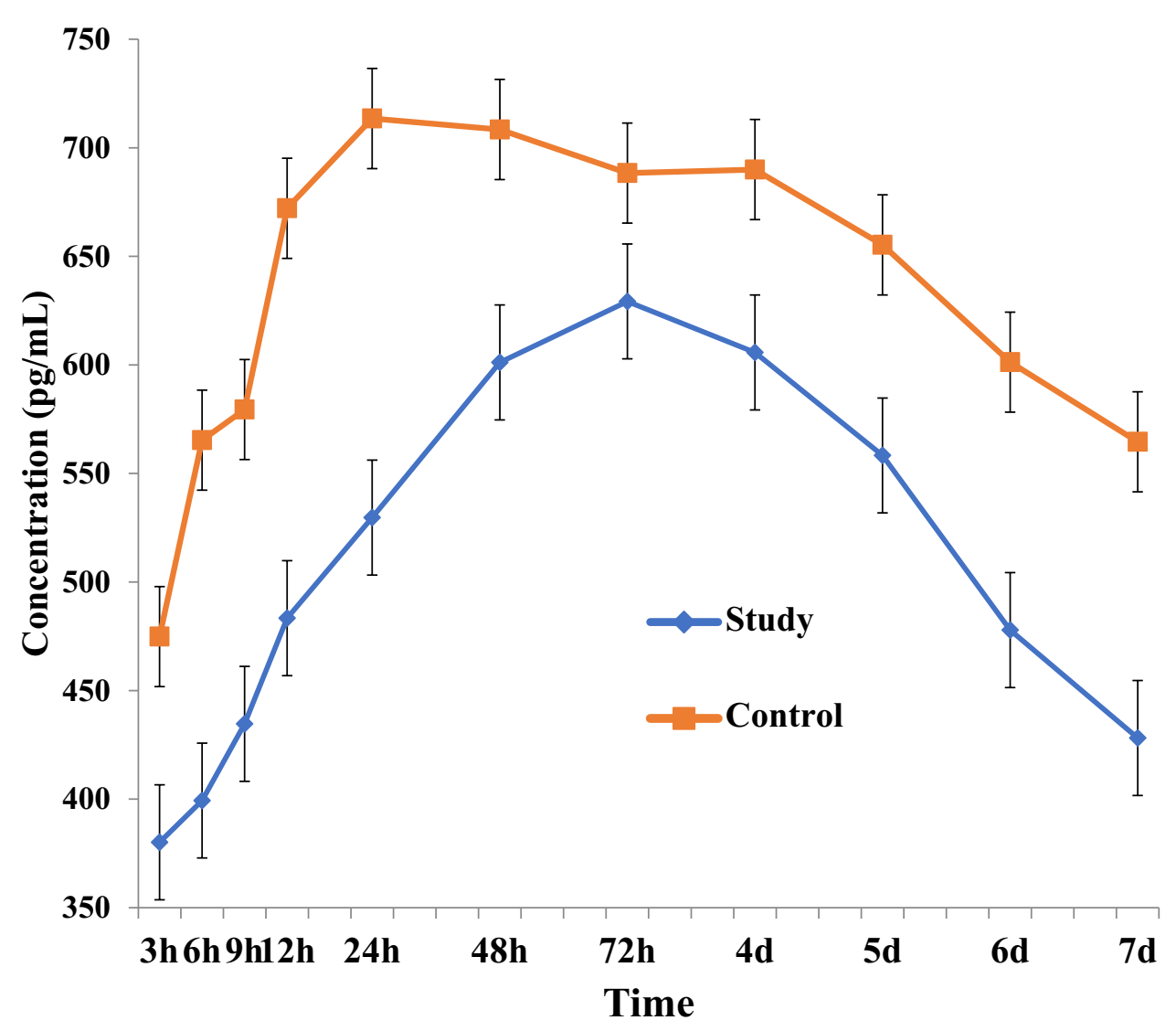

Figure 4 Changes in VEGF in the uterine exudate with time. The concentration at each time point between the study and control groups was compared, as was the concentration between the peak and the 7 th day after the operation.

which cause readhesion, which is in agreement with previous clinical results showing a readhesion rate of $62.5 \%$ after treatment with hysteroscopic adhesiolysis for severe IUA. ${ }^{2}$ Furthermore, some other factors are crucial in repairing the endometrium and incision, thereby restoring the normal function and receptivity of the endometrium.

Similar to other surgical wounds, uterine wounds after hysteroscopic adhesiolysis exude high levels of cytokines due to electrothermal effects. Among these cytokines, IL-1 $\beta$, TNF- $\alpha$, and VEGF regulate tissue damage and repair. ${ }^{10}$ Notably, TNF- $\alpha$ and VEGF are the central cytokines secreted in the early stages of inflammation. Their expression is closely related to the formation of new capillaries and the prevention of wound infections. In contrast, excessive cytokine exudation might induce excessive ECM accumulation and promote granulation tissue and fibrous scar formation. High TNF- $\alpha$ expression in wound exudates causes the release of high levels of VEGF, thereby triggering fibroblast aggregation and increased ECM synthesis. ${ }^{11}$ Moreover, TNF- $\alpha$ causes unbalanced ECM synthesis and degradation through the inhibition of the secretion of matrix metalloproteinase-9 (MMP-9), resulting in ECM accumulation and promotion of fibrous tissue hyperplasia and scar formation. ${ }^{12}$ In this study, the two aforementioned cytokines peaked on the 2nd day and decreased significantly on the 7th day after surgery. However, compared to those in the control group, the concentrations of postoperative cytokines in the study group were significantly lower.

Macrophages secrete IL- $1 \beta$ facilitated by TNF- $\alpha$ or itself, thus activating tumour necrosis factor receptor (TNFR) and IL-1 receptor 1 (R1). Here, the time required for the IL-1 $\beta$ concentration to peak was longer than the time required for TNF- $\alpha$ and VEGF to peak. ${ }^{13}$ In addition to promoting endometrial tissue repair and maintaining a balance of immune activity in the uterine cavity, IL-1 $\beta$ was found to promote a healthy intrauterine environment and normal receptivity of the endometrium. ${ }^{14}$ Although its concentration in the study group was lower than that in the control group due to inhibition of TNF- $\alpha$ and VEGF in the early stage, IL-1 $\beta$ promotes self-secretion through the IL-1 $\beta /$ IL-1R1 pathway. Moreover, its level in the study group was similar to that in the control group during the endometrial repair period $7 \mathrm{~d}$ 


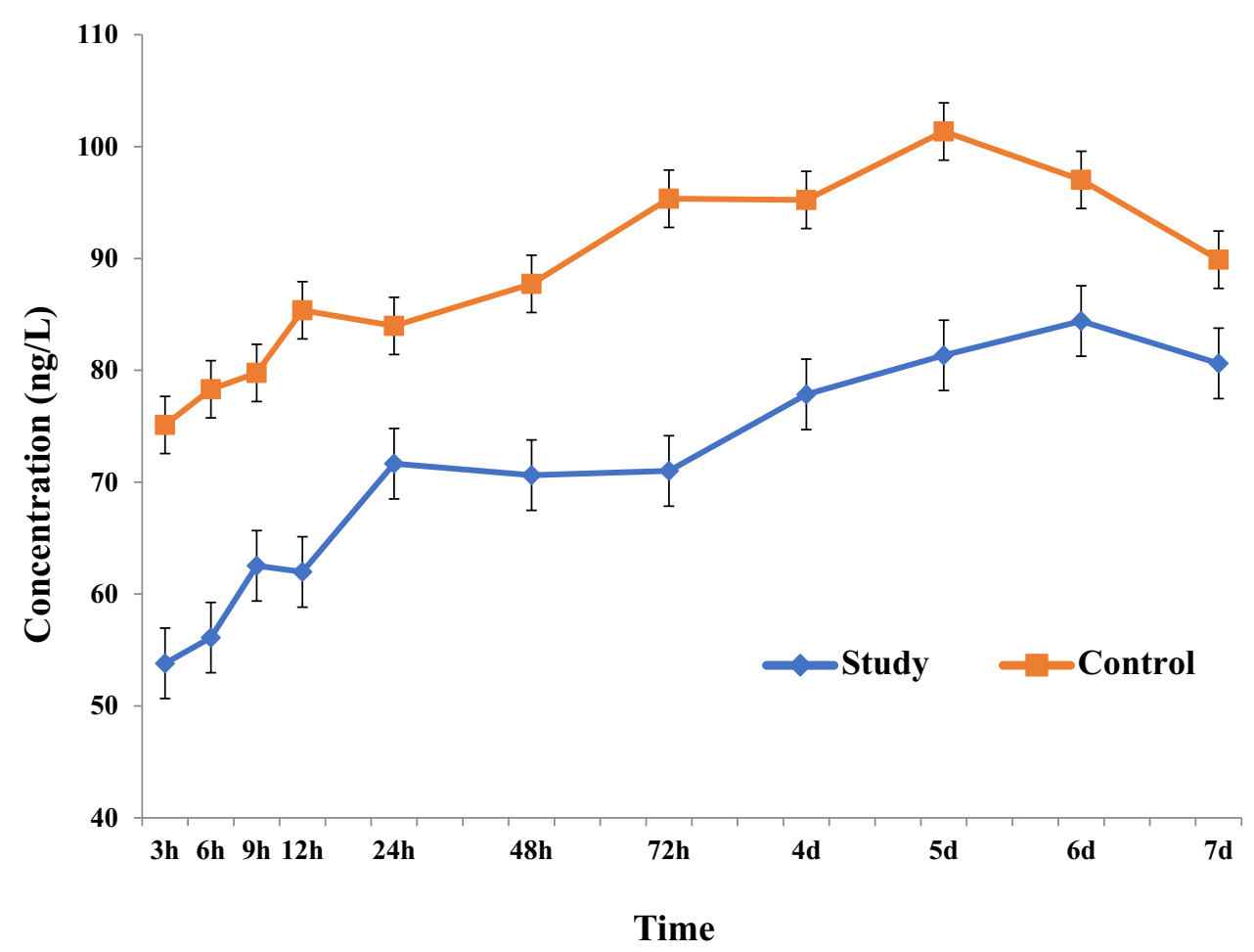

Figure 5 Changes in IL-I $\beta$ in the uterine exudate with time. The concentration at each time point between the study and control groups was compared.

A

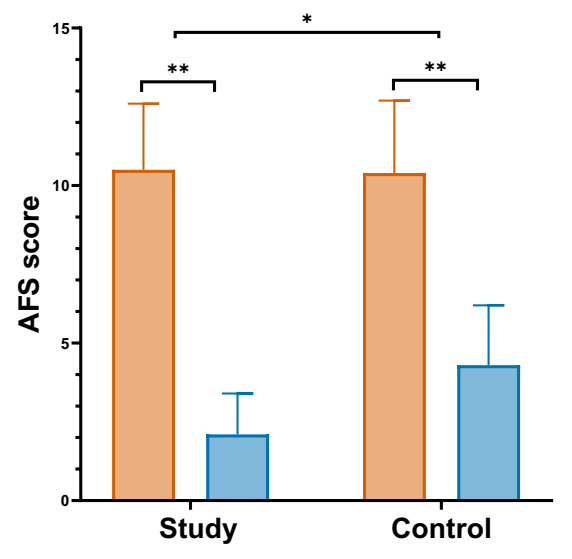

B

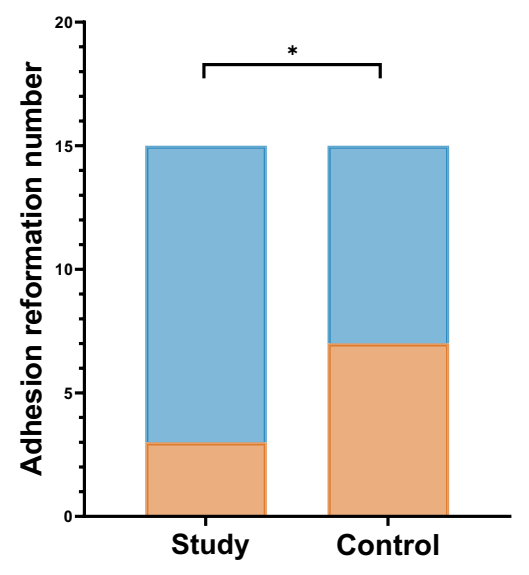

Not reformation

Reformation

Figure 6 Outcomes of second-look hysteroscopy. (A) Pre- and postoperative AFS scores between the two groups. (B) Postoperative readhesion rates between the two groups. $* P<0.05, * * P<0.01$.

after the operation. According to statistical trends, IL-1 $\beta$ in the study group was maintained for a prolonged duration to promote the endometrial repair process. Therefore, effective postoperative inhibition of adhesion-related factor exudation after hysteroscopic adhesiolysis is essential for reducing the readhesion rate and improving surgical outcomes.

Freeze-dried amnion is widely used in the medical field and contains high levels of cytokines and cytokine receptors, which promote the repair of damaged tissues. In animal studies, freeze-dried amnion grafts repaired eyelid corneal injuries in rabbits by inhibiting the expression of TNF-a and VEGF in the cornea. Further studies found that freeze-dried amnion inhibits the expression of TNF and VEGF in injured tissues, accelerates apoptosis of inflammatory cells, and reduces the inflammatory response. At the same time, freeze-dried amnion grafts can promote the migration and adhesion of corneal epithelial cells and prevent their apoptosis. Additionally, these grafts have been used in the treatment of IUAs 
due to their unique features, including the ability to reduce the release of adhesion factors and contribute to damage repair. The advantage of freeze-dried amnion grafts on intimalization after hysteroscopic adhesiolysis might be the reason why they improve reproductive prognoses. Our clinical study, which involved postoperative hysteroscopic reexamination, menstrual flow assessment, and a postoperative follow-up for an average of 14.6 months, including during pregnancy, found that postoperative readhesion scores and menstrual flow after treatment were significantly different from those before treatment, and the pregnancy rate also increased, ${ }^{15}$ indicating that freeze-dried amnion grafts can effectively improve endometrial status by preventing the occurrence of readhesions. ${ }^{16}$

Therefore, freeze-dried amnion grafts can be more effectively attached to wounds in the uterine cavity to perform their functions. While the balloon supports the anterior and posterior walls of the uterus in the uterine cavity, as a foreign body in the wound, it also stimulates the production of inflammatory cytokines. The freeze-dried amnion blocks direct contact between the rubber balloon and the wound, thereby promoting anti-inflammatory and antifibrotic responses and scar suppression. Orhue et al argued that after hysteroscopic adhesiolysis, the anterior and posterior walls of the uterus separate for at least $7 \mathrm{~d}$ before the wound can heal, whereas the function of the biological barrier in the freeze-dried amnion persists for at least $21 \mathrm{~d}$ to maintain a continuous effect in the uterine cavity. ${ }^{17}$ Furthermore, Amer et al used a balloon combined with a freeze-dried amnion graft to treat severe IUAs. ${ }^{18}$ Then, hysteroscopic exploration was performed 2 months after surgery, and the original scar tissue was obtained for examination. The basal cells of the endometrium were observed under an electron microscope and were also found on the degenerated freeze-dried amnion graft, implying that the freeze-dried amnion graft continued to function even after the removal of the balloon $7 \mathrm{~d}$ after surgery. Moreover, epithelial and mesenchymal cells derived from freeze-dried amnion have been demonstrated to survive for several weeks, which is sufficient to prevent a new cycle of fibrosis and scar healing.

Despite recent clinical studies on the application of freeze-dried amnion after hysteroscopic adhesiolysis, no consistent theoretical foundation has been established. In this study, we found that freeze-dried amnion grafts can effectively reduce wound exudation from the uterine cavity, regulate the increase in acute inflammation-related factors (TNF- $\alpha$ and VEGF), and maintain an appropriate concentration of IL-1 $\beta$ in the endometrial repair phase. Additionally, according to our follow-up results, we found significant decreases in postoperative AFS scores and postoperative readhesion rates in patients receiving freeze-dried amnion grafts compared to the control group. Thus, we believe that freeze-dried amnion grafts may improve patient outcomes by altering intrauterine cytokine concentrations.

Notably, however, the freeze-dried amnion grafts currently used in clinical practice have limitations, including morphological dimensions that do not adapt to the morphology of the uterine cavity, thus limiting their clinical application. Nevertheless, this study lays a foundation for the use of freeze-dried amnion grafts in the prevention and treatment of readhesion in severe cases of IUAs and thus provides novel strategies for preventing postoperative uterine wound readhesion after hysteroscopic adhesiolysis surgery. However, the efficacy of freeze-dried amnion in surgeries for severe IUA requires further validation by prospective, multicentre, and large-sample size studies.

According to our clinical outcomes, freeze-dried amnion grafts can effectively reduce postoperative AFS scores and readhesion rates. The duration of Foley balloon placement is an important clinical consideration. Balloon placement for too long would lead to compression and necrosis of endometrial tissues, while placing a balloon for too short a time is not effective for preventing readhesion. According to our study, removal of the balloon $7 \mathrm{~d}$ after surgery was found to be an optimal time point to effectively prevent postoperative adhesions. The time point for balloon removal after placement of a freeze-dried amnion graft may be considered to be appropriately advanced, thus reducing necrosis of the surrounding normal endometrial tissue that may result from compression.

\section{Conclusions}

This study showed that freeze-dried amnion grafts reduce the amount of uterine exudates after hysteroscopic adhesiolysis and shorten the duration of exudation. Furthermore, we discovered that freeze-dried amnion regulates the concentrations of adhesion-associated factors, including TNF- $\alpha$, VEGF, and repair-associated factors (IL-1 $\beta$ ), in uterine exudates, thereby restoring relatively low levels of these factors within a limited time. 


\section{Abbreviations}

IUAs, intrauterine adhesions; AFS, American Fertility Society; TCRA, transcervical resection of adhesions; IL-1 $\beta$, interleukin-1 beta; TNF- $\alpha$, tumour necrosis factor- $\alpha$; VEGF, vascular endothelial growth factor; ECM, extracellular matrix; ELISA, enzyme-linked immunosorbent assay; OD, optical density; SNK, Student-Newman-Keuls; MMP-9, matrix metalloproteinase-9; TNFR, tumour necrosis factor receptor.

\section{Data Sharing Statement}

The data used to support the findings of this study are available from the corresponding author upon request. These findings cannot be shared at this time as the data also forms part of an ongoing study.

\section{Ethical Approval and Informed Consent}

This study was a randomized clinical controlled trial (Trial Registration Number NCT02496052). Each participant in the study signed an informed consent form. The study was approved by the Research Ethics Committee of Beijing Obstetrics and Gynecology Hospital, where the experiments were performed (2016-KY-082-02), and was performed in accordance with the Helsinki Declaration of 1975, as revised in 2008.

\section{Author Contributions}

All authors made a significant contribution to the work reported, whether that is in the conception, study design, execution, acquisition of data, analysis and interpretation, or in all these areas; took part in drafting, revising or critically reviewing the article; gave final approval of the version to be published; have agreed on the journal to which the article has been submitted; and agree to be accountable for all aspects of the work.

\section{Funding}

This research was supported by grants from the Beijing Municipal Administration of Hospital Clinical Medicine Development of Special Funding Support (grant number ZYLX201406).

\section{Disclosure}

The authors report no conflicts of interest in this work.

\section{References}

1. Konci R, Caminsky N, Tulandi T, Dahan MH. Supplements to conventional treatment after hysteroscopic lysis of intrauterine adhesions: a systematic review. J Obstet Gynaecol Can. 2020;42(8):984-1000. doi:10.1016/j.jogc.2019.09.008

2. Di Spiezio Sardo A, Calagna G, Scognamiglio M, O'Donovan P, Campo R, De Wilde RL. Prevention of intrauterine post-surgical adhesions in hysteroscopy. A systematic review. Eur J Obstet Gynecol Reprod Biol. 2016;203:182-192. doi:10.1016/j.ejogrb.2016.05.050

3. Abudukeyoumu A, Li MQ, Xie F. Transforming growth factor- $\beta 1$ in intrauterine adhesion. Am J Reprod Immunol. 2020;84(2):e13262. doi:10.1111/ aji. 13262

4. Tao Z, Duan H. [Expression of adhesion-related cytokines in the uterine fluid after transcervical resection of adhesion]. Zhonghua Fu Chan Ke Za Zhi. 2012;47(10):734-737. Chinese.

5. Jirsova K, Jones GLA. Amniotic membrane in ophthalmology: properties, preparation, storage and indications for grafting-a review. Cell Tissue Bank. 2017;18(2):193-204. doi:10.1007/s10561-017-9618-5

6. Mohan R, Bajaj A, Gundappa M. Human amnion membrane: potential applications in oral and periodontal field. J Int Soc Prev Community Dent. 2017;7(1):15-21. doi:10.4103/jispcd.JISPCD_359_16

7. Zheng F, Zhu B, Liu Y, Wang R, Cui Y. Meta-analysis of the use of amniotic membrane to prevent recurrence of intrauterine adhesion after hysteroscopic adhesiolysis. Int J Gynaecol Obstet. 2018;143(2):145-149. doi:10.1002/ijgo.12635

8. Peng X, Li T, Zhao Y, Guo Y, Xia E. Safety and efficacy of amnion graft in preventing reformation of intrauterine adhesions. J Minim Invasive Gynecol. 2017;24(7):1204-1210. doi:10.1016/j.jmig.2017.08.005

9. Buttram VC, Gomel V, Siegler A, et al. The American Fertility Society classifications of adnexal adhesions, distal tubal occlusion, tubal occlusion secondary to tubal ligation, tubal pregnancies, müllerian anomalies and intrauterine adhesions. Fertil Steril. 1988;49(6):944-955. doi:10.1016/ S0015-0282(16)59942-7

10. Gan L, Duan H, Xu Q, et al. Human amniotic mesenchymal stromal cell transplantation improves endometrial regeneration in rodent models of intrauterine adhesions. Cytotherapy. 2017;19(5):603-616. doi:10.1016/j.jcyt.2017.02.003

11. Lennard CM, Mann EA, Sun LL, Chang AS, Bolger WE. Interleukin-1 $\beta$, Interleukin-5, interleukin-6, interleukin-8, and tumor necrosis factor- $\alpha$ in chronic sinusitis: response to systemic corticosteroids. Am J Rhinol. 2000;14(6):367-373. doi:10.2500/105065800779954329 
12. Islam MS, Ciavattini A, Petraglia F, Castellucci M, Ciarmela P. Extracellular matrix in uterine leiomyoma pathogenesis: a potential target for future therapeutics. Hum Reprod Update. 2018;24(1):59-85. doi:10.1093/humupd/dmx032

13. Chun BY, Rhiu S. Cryopreserved rabbit amniotic membrane alleviated inflammatory response and fibrosis following experimental strabismus surgery in rabbits. PLoS One. 2017;12(10):e0187058. doi:10.1371/journal.pone.0187058

14. Sadrai Z, Hajrasouliha AR, Chauhan S, Saban DR, Dastjerdi MH, Dana R. Effect of topical azithromycin on corneal innate immune responses. Invest Ophthalmol Vis Sci. 2011;52(5):2525-2531. doi:10.1167/iovs.10-5658

15. Wang X, Duan H. [Clinical evaluation of amniontic products after transcervical resection of intensive degree of intrauterine adhesions]. Zhonghua Fu Chan Ke Za Zhi. 2016;51(1):27-30. Chinese. doi:10.3760/cma.j.issn.0529-567X.2016.01.007

16. Gan L, Duan H, Sun F-Q, Xu Q, Tang Y-Q, Wang S. Efficacy of freeze-dried amnion graft following hysteroscopic adhesiolysis of severe intrauterine adhesions. Int J Gynaecol Obstet. 2017;137(2):116-122. doi:10.1002/ijgo.12112

17. Orhue AAE, Aziken ME, Igbefoh JO. A comparison of two adjunctive treatments for intrauterine adhesions following lysis. Int J Gynaecol Obstet. 2003;82(1):49-56. doi:10.1016/S0020-7292(03)00030-4

18. Amer MI, Abd-E1-Maeboud KH, AlloubA. Aminon graft as a possible source of stem cells for endometrial regeneration after lysis of severe intrauterine adhesions.MEFS. 2012;17(1):54-56.

International Journal of General Medicine

Dovepress

\section{Publish your work in this journal}

The International Journal of General Medicine is an international, peer-reviewed open-access journal that focuses on general and internal medicine, pathogenesis, epidemiology, diagnosis, monitoring and treatment protocols. The journal is characterized by the rapid reporting of reviews, original research and clinical studies across all disease areas. The manuscript management system is completely online and includes a very quick and fair peer-review system, which is all easy to use. Visit http://www.dovepress.com/testimonials.php to read real quotes from published authors.

Submit your manuscript here: https://www.dovepress.com/international-journal-of-general-medicine-journal 